\title{
A COMPARISON OF TAIL BEHAVIOUR \\ OF STOCK MARKET RETURNS
}

Krzysztof Echaust, Ph.D.

Poznań University of Economics

Al. Niepodległości 10, 61-875 Poznań, Poland

e-mail: k.echaust@ue.poznan.pl

Received 26 February 2014, Accepted 1 July 2014

\begin{abstract}
Most investors believe that left tails of the stock returns distribution are heavier than the right ones. It is a natural consequence of crashes perception as much more turbulent than the booms. Crashes develop in shorter time intervals than booms and changes of prices are significantly bigger. This paper focuses on the extreme behavior of stock market returns. The differences in the tails thickness of distribution are negligible. Its main result is that the differences between tails have been found in the clustering of extremes, especially during the crash of 2007-2009.
\end{abstract}

Keywords: fat tails, distribution, extremal dependence, extremal index.

JEL classification: G15, C4. 


\section{Introduction}

Many authors documented that financial time series do not follow the Gaussian distribution, and have much fatter tails than the latter. The earliest empirical studies come from Mandelbrot ${ }^{1}$ and Fama ${ }^{2}$. Fat tails mean large probability of extreme outcomes represented by profits and losses at the most positive or negative part of an asset or portfolio's returns distribution. From intuitive point of view it seems to be clear that the left tail must be heavier than the right one. This is due to the fact that growth trends are built over long time horizons and crashes are more violent and price changes in absolute value are much larger then. For example, the DJIA index on Black Monday fell down by $22.6 \%$ and its biggest increase since the early 1970s has been merely $11.08 \%$. Even greater disparities in the biggest changes can be seen on the example of the Hang Seng Index, where the biggest daily loss in history was $33.33 \%$, compared to $18.82 \%$ for the historical growth. There are studies indicating the presence of heavier left tails of distributions (e.g. LeBaron and Ritirupa ${ }^{3}$; Cotter $^{4}$ ). On the other hand, there are many papers which have failed to demonstrate such asymmetry between the thickness of the left and right tails (e.g. Longin ${ }^{5}$; Longin ${ }^{6}$; Jondeau and Rockinger ${ }^{7}$; Francq and Zakoïan ${ }^{8}$ ). If they are right and the left and right tail thickness is not distinguishable, there must be some other difference between them from the statistical point of view.

In this paper an attempt is made to verify the fact that the main distinction between tails is the higher dependence in extreme returns occurring in the left tail. The violence of crashes is then reflected in the clustering of extremes but not in the thickness of tails. The tail behavior of returns for twelve stock indices is compared.

The structure of this paper is as follows. In section one an estimator of tail thickness is presented, in the second section a brief description of extremal dependence is described. In the third section the test data is introduced, and in the final section the empirical results are discussed.

\section{Fat tails}

A random variable $X$ follows a heavy-tailed distribution with the index $\alpha$ if

$$
\mathbb{P}(X>x) \sim L(x) x^{-\alpha}, \text { as } x \rightarrow \infty
$$

where $L(\cdot)$ is a slowly varying function, and $\sim$ means that the ratio of both sides tends to 1 , as $x \rightarrow \infty$. Estimating the tail index $\alpha$ is not an easy task. The most common estimator of $\alpha$ is the Hill $^{9}$ estimator, which is given by 


$$
\hat{\gamma}=1 / \hat{\alpha}=1 / k \sum_{i=1}^{k} \ln x_{(n-i+1)}-\ln x_{(n-k)}
$$

where $x_{(i)}$ are the order statistics for the series, $x_{(1)} \leq x_{(2)} \leq \ldots \leq x_{(n)}, n-$ sample size, $k$ - number of observations in the tail $2 \leq k \leq n$. It is well known that if $k=k(n) \rightarrow \infty, k(n) / n \rightarrow 0$ the estimator is consistent, and asymptotically normal $\sqrt{k}(\hat{\alpha}-\alpha) \rightarrow \mathcal{N}\left(0, \alpha^{2}\right)$. The asymptotical bias and variance of the Hill estimator follow the function of $k$ :

$$
\begin{gathered}
\mathbb{E}(\hat{\gamma}(k))=\gamma+k^{\rho} \\
\operatorname{var}(\hat{\gamma}(k))=\frac{\gamma^{2}}{k}
\end{gathered}
$$

where $\rho>0$ is another parameter to estimate ${ }^{10}$.

The Hill estimator is very sensitive to the choice of the tail fraction $k$. This choice is a classical trade-off between the bias and variation. Huisman et al. ${ }^{11}$ have shown a modified Hill estimator having improved properties in comparison to a traditional Hill estimator. They assumed a specific class of distributions, called the Hall ${ }^{12}$ class with the parameter $\rho=1$. Instead of selecting one optimal $k$ to estimate the tail index, they propose to estimate a regression line:

$$
\hat{\gamma}(k)=\beta_{0}+\beta_{1} k+\varepsilon(k)
$$

where $\hat{\gamma}(k)$ is the Hill estimator for different values of $k, \varepsilon$ is a mean zero error term. The term $\beta_{1}$, in the above equation, estimates the bias, and $\beta_{0}$ gives a bias the corrected estimate of $\gamma$. This estimator is referred to as the OLS Hill estimator ${ }^{13}$.

\section{Extremal dependence}

The dependence effect in the short range behavior of extremes is measured by the extremal index $\theta$. It is a key concept of the extreme value theory for stationary processes ${ }^{14}$. Let $\left(X_{n}\right)_{n \in \mathbb{N}}$ be a strictly stationary sequence of random variables with a marginal distribution function $F$. It can be assumed that this sequence has an extremal index $\theta \in(0,1]$, that is, for each $\tau>0$ there exists a sequence of thresholds $\left(u_{n}(\tau)\right)_{n \in \mathbb{N}}$ such as $n \rightarrow \infty$

$$
n\left(1-F\left(u_{n}(\tau)\right)\right) \rightarrow \tau
$$

and 


$$
\mathbb{P}\left(M_{n} \leq u_{n}\right) \rightarrow \exp (-\theta \tau)
$$

where $M_{n}=\max \left\{X_{1}, \ldots, X_{n}\right\}$. The case of $\theta=1$ is true for both the independent and dependent process but the level of dependence is negligible at asymptotically high levels ${ }^{15}$. The extremal index measures propensity of the process to cluster at extreme levels. In this paper we focus on the block maxima model. It consists in dividing the $n$ observations into non overlapping $k$ blocks of a certain length $m$. In each block, the number of exceedances over a certain high threshold are counted, and the block estimator is then defined as

$$
\hat{\theta}=\frac{1}{m} \frac{\ln (1-M / k)}{\ln (1-N / n)}
$$

where $N$ - number of exceedances of threshold $u_{n}, M$ - number of blocks with at least one exceedance of $u_{n}{ }^{16}$.

\section{The data}

Our research is performed for daily closing prices of twelve equity market indices obtained for the period between 2007 and 2013. The following region leading indices are chosen: S\&P500, Bovespa, Buenos, Nikkei225, Hang Seng, DAX, CAC40, FTSE100, SMI, EOE, BUX and WIG20. The calculations are based on the percentage logarithmic returns $r_{t}=100 \cdot \ln \left(p_{t} / p_{t-1}\right)$. The Table 1 presents some relevant summary statistics.

Table 1. Descriptive statistics of the return series

\begin{tabular}{|l|c|c|c|c|c|c|c|c|}
\hline \multicolumn{1}{|c|}{ Index } & Nobs & Min & Max & Mean & Median & St.dev. & Skewness & Kurtosis \\
\hline SP500 & 1760 & -9.47 & 10.96 & 0.02 & 0.08 & 1.48 & -0.302 & 8.492 \\
\hline BOVESPA & 1729 & -12.10 & 13.68 & 0.01 & 0.08 & 1.91 & -0.017 & 6.362 \\
\hline BUENOS & 1713 & -12.95 & 10.43 & 0.05 & 0.10 & 2.01 & -0.569 & 4.946 \\
\hline NIKKEI & 1713 & -12.11 & 13.23 & 0.00 & 0.05 & 1.74 & -0.571 & 7.643 \\
\hline HANGSENG & 1725 & -13.58 & 13.41 & 0.01 & 0.06 & 1.83 & 0.067 & 7.294 \\
\hline DAX & 1780 & -7.90 & 10.80 & 0.02 & 0.08 & 1.55 & 0.070 & 6.114 \\
\hline CAC40 & 1792 & -9.47 & 10.59 & -0.01 & 0.02 & 1.63 & 0.098 & 5.531 \\
\hline FTSE100 & 1774 & -9.27 & 9.38 & 0.00 & 0.03 & 1.38 & -0.115 & 6.737 \\
\hline SMI & 1758 & -8.11 & 10.79 & 0.00 & 0.04 & 1.28 & 0.043 & 7.081 \\
\hline EOE & 1792 & -9.59 & 10.03 & -0.01 & 0.04 & 1.54 & -0.143 & 7.578 \\
\hline BUX & 1746 & -12.65 & 13.18 & -0.02 & 0.01 & 1.80 & -0.027 & 6.766 \\
\hline WIG20 & 1751 & -8.44 & 8.15 & -0.02 & 0.02 & 1.65 & -0.241 & 2.911 \\
\hline
\end{tabular}

Source: own calculations. 
The difference between the absolute minimum and the maximum is very small. The smallest spreads between maxima and minima are observed for S\&P500 and the European indices excluding BUX. Likewise, standard deviations are the lowest for those indices indicating their relative stability. In all cases excess kurtosis shows too many realizations clustering at the tails of distributions relative to normal distribution. It is confirmed by the Jarque-Bera test which rejected normality for all indices. The lowest spread and kurtosis is obtained for Polish WIG20. Surprisingly, it is the index listed on the market which is classified as an emerging market and thus characterized by a high extreme risk.

\section{Empirical results}

Fat tails of empirical distributions are the result of the occurrence of extreme returns. Many empirical studies on a financial time series indicate that the tail index is greater than two, confirming the existence of variance, and very seldom it exceeds five ${ }^{17}$. Note that the smaller the value of $\alpha$, the fatter the tail of the distribution. In the first point the characteristics of distribution tails will be compared for the entire period of 7 years. However, in the second point the results will be presented separately for the financial crisis of 2007-2009 and for the economic boom that followed later.

\subsection{Entire period of 2007-2013}

In Table 2 we present the OLS estimates of the left and right tail indices. The results are consistent with the above mentioned ones. The values of the tail index lie in the interval $(2,4)$. When we compare the tail thickness between left and right tails it is difficult to find any differences.

Table 2. Tail indices and standard errors in parentheses

\begin{tabular}{|l|c|c|}
\hline \multicolumn{1}{r|}{ Index } & Left & Right \\
\hline SP500 & 2 & 3 \\
\hline BOVESPA & $2.625(0.015)$ & $2.620(0.015)$ \\
\hline BUENOS & $2.917(0.013)$ & $2.898(0.006)$ \\
\hline NIKKEI & $2.781(0.016)$ & $3.221(0.022)$ \\
\hline HANGSENG & $2.674(0.006)$ & $3.363(0.009)$ \\
\hline DAX & $3.104(0.026)$ & $2.885(0.006)$ \\
\hline CAC40 & $3.006(0.033)$ & $2.931(0.010)$ \\
\hline FTSE100 & $3.068(0.021)$ & $2.935(0.007)$ \\
\hline SMI & $2.826(0.015)$ & $2.818(0.007)$ \\
\hline
\end{tabular}




\begin{tabular}{|l|c|c|}
\hline \multicolumn{1}{|c|}{1} & 2 & 3 \\
\hline EOE & $2.697(0.013)$ & $2.755(0.007)$ \\
\hline BUX & $2.919(0.007)$ & $2.927(0.014)$ \\
\hline WIG20 & $3.049(0.019)$ & $3.443(0.029)$ \\
\hline
\end{tabular}

Source: own calculations.

The biggest differences in tail thickness are noticeable in the case of Buenos and Nikkei, where the left tail is thicker than the right one. However, in general, we cannot state that negative extreme returns are more frequent than the positive ones, but their behaviour is very similar. As an example see Figure 1 for S\&P500 index where asymmetry in the tails is not visible.

The key analysis concerns the issue of dependence in extreme returns. We verify a hypothesis that the perception of left tails being heavier than the right ones could be due to more intense clustering of events in the left tail. Such hypothesis was negatively verified by Jondeau and Rockinger ${ }^{18}$ who stated that clustering of news cannot be the reason why investors consider left tails to be heavier than right tails.

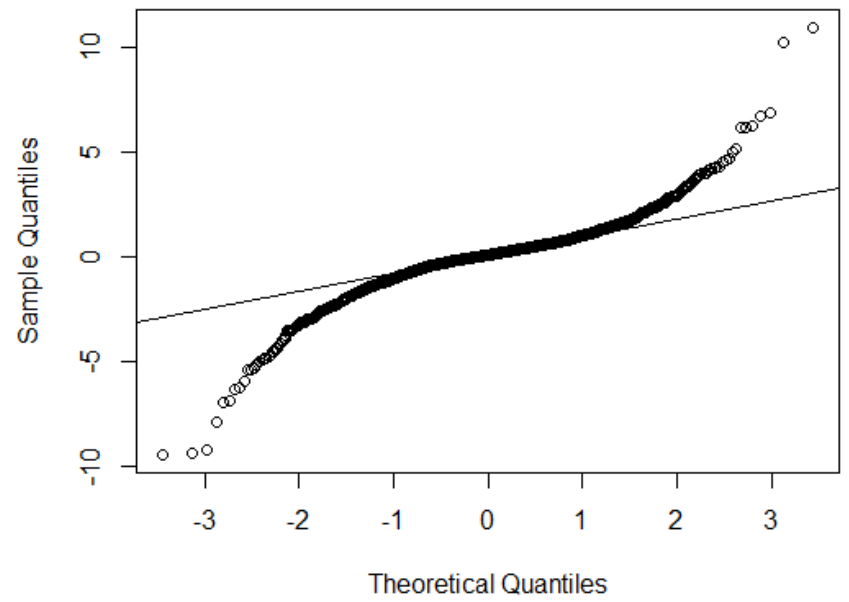

Fig. 1. QQ plot for S\&P500 Composite Index

Source: own calculations.

Similarly to their work, in this analysis, one week blocks of returns to calculate extremal index are assumed. As it is known the strength of dependence decreases as we move into the tail regions of distribution, even if the returns are highly dependent in the center of the distribution ${ }^{19}$. For that purpose we estimate an extremal index for various levels of the threshold given as quantiles of the empirical distribution. The results are presented in Table 3. By the bold type 
those cases were marked where extremal index calculated for the left tail is higher than for the right one. It means that extremes in the right tail cluster more than in the left one. Such contingency occurs only for Nikkei and twice for BUX index. In all the other considered cases extremal dependence is higher in the left tail of distribution. Such property can be recognized as typical.

Table 3. Extremal index for various threshold levels

\begin{tabular}{|c|c|c|c|c|c|c|c|c|}
\hline Quantile $u_{n}$ & \multicolumn{2}{|c|}{ SP500 } & \multicolumn{2}{c|}{ BOVESPA } & \multicolumn{2}{c|}{ BUENOS } & \multicolumn{2}{c|}{ NIKKEI } \\
\hline 0.95 & 0.86 & 0.93 & 0.81 & 0.96 & 0.83 & 0.91 & $\mathbf{0 . 8 6}$ & $\mathbf{0 . 8 3}$ \\
\hline 0.96 & 0.84 & 0.89 & 0.80 & 0.98 & 0.82 & 0.91 & $\mathbf{0 . 8 3}$ & $\mathbf{0 . 8 2}$ \\
\hline 0.97 & 0.77 & 0.92 & 0.79 & 0.97 & 0.84 & 0.93 & $\mathbf{0 . 8 2}$ & $\mathbf{0 . 7 8}$ \\
\hline 0.98 & 0.77 & 0.86 & 0.82 & 0.96 & 0.79 & 0.93 & $\mathbf{0 . 8 5}$ & $\mathbf{0 . 8 2}$ \\
\hline 0.99 & 0.79 & 0.91 & 0.88 & 0.93 & 0.83 & 0.98 & $\mathbf{0 . 8 8}$ & $\mathbf{0 . 7 8}$ \\
\hline 0.995 & 0.90 & 0.90 & 0.86 & 0.94 & 0.84 & 1.00 & $\mathbf{0 . 8 4}$ & $\mathbf{0 . 7 6}$ \\
\hline Quantile $u_{n}$ & \multicolumn{2}{|c|}{ HANGSENG } & \multicolumn{2}{|c|}{ DAX } & \multicolumn{2}{|c|}{ CAC } & \multicolumn{2}{c|}{ FTSE100 } \\
\hline 0.95 & 0.78 & 0.82 & 0.87 & 0.95 & 0.88 & 0.96 & 0.80 & 0.92 \\
\hline 0.96 & 0.75 & 0.83 & 0.88 & 0.96 & 0.82 & 1.00 & 0.84 & 0.94 \\
\hline 0.97 & 0.76 & 0.83 & 0.90 & 0.92 & 0.77 & 0.97 & 0.84 & 0.89 \\
\hline 0.98 & 0.76 & 0.92 & 0.89 & 0.89 & 0.82 & 0.93 & 0.83 & 0.86 \\
\hline 0.99 & 0.73 & 0.91 & 0.90 & 1.00 & 0.87 & 0.97 & 0.83 & 0.83 \\
\hline 0.995 & 0.67 & 0.78 & 0.78 & 1.00 & 0.83 & 1.00 & 0.78 & 0.86 \\
\hline Auantile $u_{n}$ & \multicolumn{2}{|c|}{ SMI } & \multicolumn{2}{|c|}{ EOE } & & BUX & \multicolumn{2}{|c|}{ WIG20 } \\
\hline 0.95 & 0.81 & 0.89 & 0.84 & 0.96 & 0.87 & 0.90 & 0.86 & 0.94 \\
\hline 0.96 & 0.81 & 0.84 & 0.81 & 0.95 & 0.84 & 0.86 & 0.83 & 0.96 \\
\hline 0.97 & 0.75 & 0.83 & 0.77 & 0.95 & $\mathbf{0 . 8 8}$ & $\mathbf{0 . 8 4}$ & 0.82 & 1.00 \\
\hline 0.98 & 0.71 & 0.77 & 0.79 & 0.93 & $\mathbf{0 . 9 8}$ & $\mathbf{0 . 8 3}$ & 0.86 & 1.00 \\
\hline 0.99 & 0.73 & 0.78 & 0.81 & 0.92 & 0.91 & 0.91 & 0.91 & 1.00 \\
\hline 0.995 & 0.76 & 0.76 & 0.83 & 1.00 & 0.91 & 0.91 & 0.91 & 1.00 \\
\hline
\end{tabular}

Source: own calculations.

Especially evident asymmetry in clustering can be noticed for the CAC40, EOE and WIG20 indices. Because the left tail of CAC40 distribution is not heavier than the right tail, it cannot be concluded that higher dependence in the left tail is connected with a higher tail thickness. In further part of this work we try to answer as well whether the tail behaviour differs during crashes and booms on stock markets.

\subsection{Crash and boom period}

In the entire period of seven years, two sub-periods have been distinguished. First, it is a credit crunch period: 19 July 2007 - 9 March 2009. The initial date is the day when S\&P500 reached its maximum after the four-year-period of permanent growths. The final date is the index 
minimum, half a year after the bankruptcy of Lehman Brothers. The other sub-period covers the dates of 10 March 2009 - 25 October 2010. It was the time of growths on financial markets which was followed the crash period. The final date of that sub-period has been especially chosen so that the periods of crisis and boom can be equally long. Those two sub-periods at S\&P500 are presented in Figure 2.

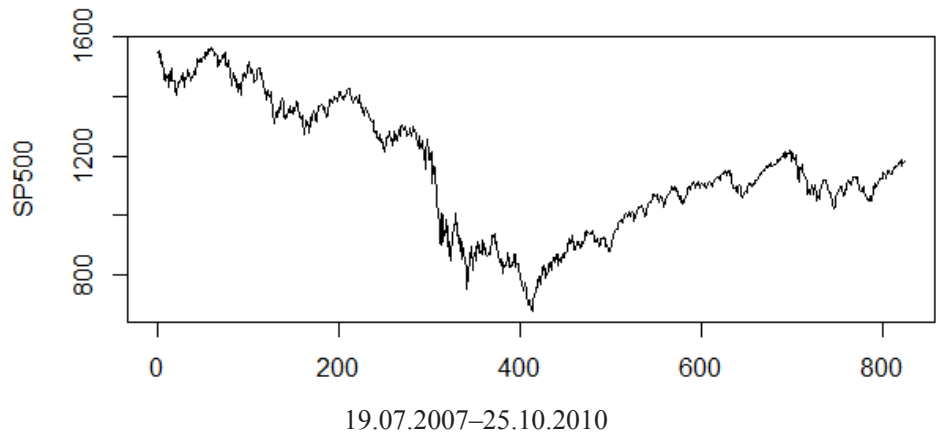

Fig. 2. S\&P500 Composite Index in the period of crisis and boom

Source: Stooq.pl (10.02.2014).

In most cases the decrease of indices in the time of crisis was much deeper than the increase during a boom. The situation at S\&P500 index is presented in Figure 2. The scale of growths and drops of individual indices in both sub-periods is shown in Table 4.

Table 4. Log-returns in times of crisis and boom (\%)

\begin{tabular}{|l|c|c|}
\hline \multicolumn{1}{|c|}{ Index } & Crash & Boom \\
\hline SP500 & -83 & 56 \\
\hline BOVESPA & -46 & 64 \\
\hline BUENOS & -88 & 112 \\
\hline NIKKEI & -94 & 28 \\
\hline HANGSENG & -71 & 73 \\
\hline DAX & -77 & 59 \\
\hline CAC40 & -88 & 43 \\
\hline FTSE100 & -63 & 48 \\
\hline SMI & -76 & 41 \\
\hline EOE & -103 & 54 \\
\hline BUX & -110 & 85 \\
\hline WIG20 & -98 & 60 \\
\hline
\end{tabular}

Source: own calculations. 
The difference in the dynamics of growths and drops in both periods translates into the tail thickness of the return rate distribution. The tail indices in the crisis time are lower than in the time of boom so the returns distributions in the time of crisis have thicker tails than in the time of growth (see Table 5). However, when the values of left and right tail indices are compared in individual periods, it is hard to notice any differences. The biggest difference between the indices of left and right tail is noticeable in case of Hang Seng index in the time of crisis. Interestingly, the right tail of distribution is then thicker than the left one, which, additionally, is hard to explain. From the statistical point of view it is not possible to prove that left tails are heavier than right ones.

Table 5. Tail indices and standard errors (in parentheses) during the crash and boom

\begin{tabular}{|l|c|c|c|c|}
\cline { 2 - 5 } \multicolumn{1}{c|}{} & \multicolumn{2}{c|}{ Crash } & \multicolumn{2}{c|}{ Boom } \\
\hline Index & left & right & left & right \\
\hline SP500 & $2.825(0.037)$ & $2.552(0.049)$ & $3.384(0.069)$ & $3.143(0.038)$ \\
\hline BOVESPA & $3.349(0.040)$ & $2.591(0.016)$ & $3.598(0.104)$ & $3.162(0.027)$ \\
\hline BUENOS & $2.667(0.045)$ & $2.373(0.013)$ & $3.162(0.027)$ & $3.004(0.021)$ \\
\hline NIKKEI & $2.690(0.039)$ & $2.824(0.021)$ & $4.843(0.139)$ & $3.812(0.047)$ \\
\hline HANGSENG & $4.006(0.043)$ & $2.753(0.023)$ & $4.107(0.085)$ & $3.466(0.034)$ \\
\hline DAX & $2.705(0.057)$ & $2.242(0.010)$ & $3.425(0.059)$ & $4.062(0.057)$ \\
\hline CAC40 & $2.858(0.032)$ & $2.455(0.018)$ & $3.376(0.069)$ & $3.793(0.030)$ \\
\hline FTSE100 & $2.730(0.026)$ & $2.630(0.017)$ & $3.649(0.124)$ & $3.225(0.027)$ \\
\hline SMI & $2.998(0.026)$ & $2.379(0.014)$ & $3.755(0.104)$ & $3.626(0.025)$ \\
\hline EOE & $2.663(0.036)$ & $2.224(0.020)$ & $3.420(0.070)$ & $3.694(0.023)$ \\
\hline BUX & $2.405(0.016)$ & $2.317(0.016)$ & $4.192(0.078)$ & $3.720(0.051)$ \\
\hline WIG20 & $3.107(0.057)$ & $3.587(0.058)$ & $3.856(0.070)$ & $3.286(0.034)$ \\
\hline
\end{tabular}

Source: own calculations.

It is different in the case of the dependencies of extremal returns. The values of extremal index for times of crash and boom are presented in Tables 6 and 7. In the crisis period these values are significantly lower than in the boom time. This implies that the clustering of extremes in the time of crisis is much more intense than in the time of stock exchange growths. In the time of growth the values of the extremal index are close to 1, so the extremes appear as approximately independent, without the clustering effect. Meanwhile, in the crisis period the clustering of extremes is clearly noticeable. Due to the expectations, the intensity of clustering in the left tails is higher than in the right one then. These results prove that the extremal drops occur in runs, so the investors' losses are accumulated. Therefore in investors' conscience a belief is created that left tails must be thicker than the right ones, though the actual difference is only in the extremal returns clustering. 
Table 6. Extremal index during the crash for various threshold levels

\begin{tabular}{|c|c|c|c|c|c|c|c|c|}
\hline Quantile $u_{n}$ & \multicolumn{2}{|c|}{ SP500 } & \multicolumn{2}{c|}{ BOVESPA } & \multicolumn{2}{c|}{ BUENOS } & \multicolumn{2}{c|}{ NIKKEI } \\
\hline 0.95 & 0.70 & 0.91 & 0.83 & 0.88 & 0.71 & 0.83 & 0.81 & 0.81 \\
\hline 0.96 & 0.73 & 0.91 & 0.89 & 0.89 & 0.75 & 0.82 & 0.80 & 0.87 \\
\hline 0.97 & 0.77 & 0.93 & 0.81 & 0.98 & 0.89 & 0.89 & 0.78 & 0.78 \\
\hline 0.98 & 0.85 & 0.85 & 0.80 & 0.93 & 0.80 & 0.93 & 0.64 & 0.77 \\
\hline 0.99 & 0.88 & 0.88 & 1.00 & 1.00 & 0.82 & 1.00 & 0.76 & 0.76 \\
\hline 0.995 & $\mathbf{1 . 0 0}$ & $\mathbf{0 . 8 2}$ & 1.00 & 1.00 & 0.67 & 1.00 & 1.00 & 1.00 \\
\hline Quantile $u_{n}$ & \multicolumn{2}{|c|}{ HANGSENG } & \multicolumn{2}{|c|}{ DAX } & \multicolumn{2}{|c|}{ CAC } & \multicolumn{2}{c|}{ FTSE100 } \\
\hline 0.95 & 0.75 & 0.95 & 0.82 & 0.98 & 0.86 & 0.98 & 0.79 & 1.00 \\
\hline 0.96 & 0.89 & 0.89 & 0.81 & 0.99 & 0.85 & 0.99 & 0.83 & 0.97 \\
\hline 0.97 & $\mathbf{0 . 9 0}$ & $\mathbf{0 . 8 1}$ & 0.72 & 1.00 & 0.79 & 0.94 & 0.83 & 0.91 \\
\hline 0.98 & 0.80 & 0.80 & 0.69 & 1.00 & 0.69 & 0.88 & 0.83 & 0.83 \\
\hline 0.99 & 0.82 & 0.82 & 0.77 & 1.00 & 0.64 & 0.91 & 0.85 & 1.00 \\
\hline 0.995 & 0.67 & 0.67 & 0.88 & 1.00 & 0.68 & 1.00 & 0.76 & 1.00 \\
\hline Quantile $u_{n}$ & \multicolumn{2}{|c|}{ SMI } & \multicolumn{2}{|c|}{ EOE } & \multicolumn{2}{|c|}{ BUX } & \multicolumn{2}{|c|}{ WIG20 } \\
\hline 0.95 & 0.69 & 0.89 & 0.75 & 0.93 & $\mathbf{0 . 8 8}$ & $\mathbf{0 . 8 3}$ & 0.72 & 0.93 \\
\hline 0.96 & 0.66 & 0.88 & 0.67 & 0.87 & $\mathbf{0 . 8 9}$ & $\mathbf{0 . 8 2}$ & 0.81 & 0.87 \\
\hline 0.97 & 0.82 & 0.82 & 0.70 & 0.94 & 0.81 & 0.81 & 0.87 & 0.87 \\
\hline 0.98 & 0.89 & 0.89 & 0.75 & 1.00 & $\mathbf{0 . 8 0}$ & $\mathbf{0 . 6 8}$ & $\mathbf{0 . 9 7}$ & $\mathbf{0 . 8 8}$ \\
\hline 0.99 & $\mathbf{0 . 9 3}$ & $\mathbf{0 . 8 0}$ & 0.77 & 1.00 & 0.82 & 0.82 & 0.91 & 0.91 \\
\hline 0.995 & 0.88 & 0.88 & 0.68 & 1.00 & $\mathbf{1 . 0 0}$ & $\mathbf{0 . 6 7}$ & 0.85 & 1.00 \\
\hline
\end{tabular}

Source: own calculations.

Table 7. Extremal index during the boom for various threshold levels

\begin{tabular}{|c|c|c|c|c|c|c|c|c|c|}
\hline Quantile $u_{n}$ & \multicolumn{2}{|c|}{ SP500 } & \multicolumn{2}{c|}{ BOVESPA } & \multicolumn{2}{c|}{ BUENOS } & \multicolumn{2}{c|}{ NIKKEI } \\
\hline 0.95 & 1.00 & 0.91 & $\mathbf{0 . 9 2}$ & $\mathbf{0 . 9 7}$ & $\mathbf{0 . 8 7}$ & $\mathbf{0 . 9 3}$ & 0.93 & 0.77 \\
\hline 0.96 & 1.00 & 0.91 & 1.00 & 0.98 & $\mathbf{0 . 8 7}$ & $\mathbf{1 . 0 0}$ & 1.00 & 0.75 \\
\hline 0.97 & 1.00 & 0.93 & 1.00 & 1.00 & 0.97 & 0.97 & 1.00 & 0.73 \\
\hline 0.98 & 1.00 & 0.85 & 1.00 & 0.96 & $\mathbf{0 . 9 1}$ & $\mathbf{1 . 0 0}$ & 0.97 & 0.69 \\
\hline 0.99 & 1.00 & 0.88 & 1.00 & 1.00 & 1.00 & 1.00 & 0.91 & 0.77 \\
\hline 0.995 & 1.00 & 0.82 & 1.00 & 1.00 & 1.00 & 1.00 & 1.00 & 0.68 \\
\hline Quantile $u_{n}$ & \multicolumn{2}{|c|}{ HANGSENG } & \multicolumn{2}{|c|}{ DAX } & \multicolumn{2}{|c|}{ CAC } & \multicolumn{2}{c|}{ FTSE100 } \\
\hline 0.95 & 1.00 & 0.73 & 1.00 & 1.00 & $\mathbf{0 . 9 3}$ & $\mathbf{0 . 9 8}$ & 1.01 & 0.95 \\
\hline 0.96 & 0.97 & 0.71 & 1.00 & 1.00 & 0.99 & 0.99 & 0.97 & 0.90 \\
\hline 0.97 & 0.91 & 0.75 & 1.00 & 1.00 & 1.00 & 0.94 & 0.99 & 0.83 \\
\hline 0.98 & 0.94 & 0.94 & 1.00 & 0.96 & 1.00 & 0.88 & 0.94 & 0.83 \\
\hline 0.99 & 1.0 & 1.0 & 1.00 & 0.88 & 1.00 & 0.77 & 0.85 & 0.85 \\
\hline 0.995 & 1.0 & 1.0 & 1.00 & 1.00 & 1.00 & 0.85 & 1.00 & 0.76 \\
\hline Quantile $u_{n}$ & \multicolumn{3}{|c|}{ SMI } & \multicolumn{2}{|c|}{ EOE } & & BUX & \multicolumn{2}{|c|}{ WIG20 } \\
\hline 0.95 & 0.91 & 0.91 & $\mathbf{0 . 9 3}$ & $\mathbf{1 . 0 0}$ & 1.00 & 0.86 & 0.98 & 0.93 \\
\hline 0.96 & 0.91 & 0.91 & $\mathbf{0 . 9 3}$ & $\mathbf{0 . 9 9}$ & 0.98 & 0.91 & 0.99 & 0.93 \\
\hline 0.97 & $\mathbf{0 . 9 3}$ & $\mathbf{1 . 0 0}$ & 0.94 & 0.94 & 1.00 & 0.85 & $\mathbf{0 . 9 4}$ & $\mathbf{1 . 0 0}$ \\
\hline 0.98 & 0.96 & 0.96 & 0.97 & 0.97 & 1.00 & 0.96 & 1.00 & 1.00 \\
\hline 0.99 & 0.88 & 0.88 & 1.00 & 1.00 & 1.00 & 0.88 & 1.00 & 1.00 \\
\hline 0.995 & 1.00 & 0.82 & 1.00 & 1.00 & 1.00 & 1.00 & 1.00 & 1.00 \\
\hline
\end{tabular}

Source: own calculations. 


\section{Conclusions}

Statistical properties of the returns distributions are investigated due to investors' perception which leads to the assumption that left tails are heavier than the right ones. From the statistical point of view, such an argument is not acceptable. The fact which tail of the distribution is heavier depends on the considered market. Besides, differences in the thickness of both tails are usually negligible. Even if we compare the tails of distribution in the crash and boom period the differences are not visible although during the crash both tails are heavier than during the boom. Differences in the tail behaviour are rather the result of the clustering of extremes. Such phenomena are found in almost all stock market returns considered in this paper. The dependence between the extreme returns increases during crashes and weakens during booms.

\section{Notes}

1 Mandelbrot (1963).

2 Fama (1965).

3 LeBaron, Ritirupa (2005).

4 Cotter (2006).

5 Longin (1996).

6 Longin (2001).

7 Jondeau, Rockinger (2003).

8 Francq, Zakoïan (2013).

9 Hill (1975).

${ }^{10}$ LeBaron (2008).

11 Huisman et al. (2001).

12 Hall (1990).

${ }^{13}$ Huisman at al. (2001); LeBaron (2008).

${ }^{14}$ Leadbetter, Lindgren, Rootzen (1983).

15 Beirlant at al. (2004); Coles (2001).

${ }^{16}$ Embrechts at al. (1999).

17 Ibidem; Longin (1996); Jansen, de Vries (1991); Loretan, Phillips (1994); Francq, Zakoïan (2013).

18 Jondeau, Rockinger (2003).

19 Danielsson (2011). 


\section{References}

Beirlant, J., Goegebeur, Y., Segers, J. \& Teugels, J. (2004). Statistics of Extremes. Theory and Applications. Chichester: Wiley.

Coles, S.G. (2001). An Introduction to Statistical Modeling of Extreme Values. London: Springer.

Cotter, J. (2006). Extreme value estimation of boom and crash statistics. The European Journal of Finance, 12 (6-7), 553-566.

Danielsson, J. (2011). Financial risk forecasting: the theory and practice of forecasting market risk with implementation in $R$ and Matlab. Chichester: Wiley.

Embrechts, P., Klüppelberg, C. \& Mikosch, T. (1999). Modelling Extremal Events for Insurance and Finance. New York: Springer.

Embrechts, P., Sidney, I., Resnick, S.I. \& Samorodnitsky, G. (1999). Extreme Value Theory as a Risk Management Tool. North American Actuarial Journal, 3 (2), 30-41.

Fama, E. (1965). The Behavior of Stock Market Prices. Journal of Business, 38, 34-105.

Francq, C. \& Zakoïan, J.M. (2013). Estimating the Marginal Law of a Time Series with Applications to Heavy Tailed Distributions. Journal of Business \& Economic Statistics, 31, $412-425$.

Hall, P. (1990). Using the bootstrap to estimate mean squared error and select smoothing parameter in nonparametric problems. Journal of Multivariate Analysis, 32, 177-203.

Hill, B.M. (1975). A simple general approach to inference about the tail of a distribution. Annals of Statistics, 3, 1163-1173.

Huisman, R., Koedijk, K.G., Kool, C.J.M. \& Palm, F. (2001). Tail-index estimates in small samples. Journal of Business and Economic Statistics, 19, 208-216.

Jondeau, E. \& Rockinger, M. (2003). Testing for differences in the tails of stock-market returns. Journal of Empirical Finance, 10, 559-581.

Jansen, D. \& de Vries, C. (1991). On the frequency of large stock market returns: putting booms and busts into perspective. Review of Economics and Statistics, 23, 18-24.

Laedbetter, M.R., Lindgren, G. \& Rootzen, H. (1983). Extremes and Related Properties of Random Sequences and Processes. New York: Springer Verlag.

LeBaron, B. \& Ritirupa, S. (2005). Extreme value theory and fat tails in equity markets. Computing in Economics and Finance, 140, Society for Computational Economics.

LeBaron, B. (2008). Robust properties of stock returns tails. International Business School, Brandeis University.

Longin, F.M. (1996). The asymptotic distribution of extreme stock market returns. Journal of Business, 69, 383-408. 
Longin, F. (2001). Stock market crashes: Some quantitative results based on extreme value theory. Derivatives Use, Trading and Regulation, 7, 197-205.

Loretan, M. \& Phillips, P.C.B. (1994). Testing the covariance stationarity of heavy-tailed time series. Journal of Empirical Finance, 1, 211-248.

Mandelbrot, B. (1963). The Variation of Certain Speculative Prices. Journal of Business, 36, 394-419. 